\title{
Modeling Study on Dynamic Spectrum Sharing System Under Interference Temperature Constraints in Underground Coal Mines
}

\author{
Chen Guizhen ${ }^{*}, 1$, Ding Enjie ${ }^{2}$, Wang Gang ${ }^{2}$ and Xue Xue ${ }^{1}$ \\ ${ }^{I}$ School of Information and Electrical Engineering, China University of Mining and Technology, Xuzhou, Jiangsu, \\ 221008, P.R. China \\ ${ }^{2}$ IOT/Perception Mine Research Center, China University of Mining and Technology, Xuzhou, Jiangsu, 221008, P.R. \\ China
}

\begin{abstract}
This paper proposes a dynamic spectrum access system for underground wireless communication- $-\mathrm{a}$ dynamic spectrum sharing system under interference temperature constrains. It can make the best of spectrum resources and improve the utilization efficiency. Then, a multi-dimensional Markov chain is used to model the system. On the basis, the secondary users' performance under interference temperature constrains is obtained. As two important performance indexes to measure secondary users' performance, cognitive users' interrupting probability and blocking probability are calculated. Finally, cognitive users' performance under different users' access is analyzed, and the performances in dynamic spectrum access system and overlay access system are compared. Simulation results indicate that the dynamic spectrum sharing access system under interference temperature constrains is superior to the overlay access system and helpful to improve the spectrum sharing system in coal mines.
\end{abstract}

Keywords: Cognitive users blocking probability, cognitive users interrupting probability, dynamic spectrum access, interference temperature.

\section{INTRODUCTION}

Coal mine tunnels are characterized by various shapes, sizes, corners, branches and inclinations. However, in traditional wireless communication system, the parameters of carrier frequency and modulation are generally fixed, which is hard to adapt to the tunnel environment with variable transmission characteristics. Especially on the Internet of Things (IOT), with the wide use of intelligent cap lamps with wireless transmission function, wireless $\mathrm{AP}$, as well as the establishment of unified monitoring platform based on wire and wireless integrated access, the mine communication environment becomes even more complicated. Previous studies $[1,2]$ presented open architecture of mines on Internet of Things and listed many concurrent wireless communication methods in mines. With the ability of sensing the environment around the tunnel, cognitive radio technology can be used to solve the above problems in coal mines communication. Through self-learning intelligent ways, it can change the transmission parameters of transmission power, carrier frequency and modulation in real time to adapt to the changes in the operating environment. For this, dynamic spectrum sharing mechanism of communication system in tunnels needs to be studied to let the users better work

*Address correspondence to this author at the School of Information and Electrical Engineering, China University of Mining and Technology, Xuzhou, Jiangsu, P.R. China; Tel: +86 51683590832 ;

E-mail: gzhchxz@126.com together and improve the reliability of signal transmission by adaptively changing its parameters.

The advantages of cognitive radio technology in underground wireless communication were analyzed [3], and presented in that the traditional underground wireless communication way that once the wireless communication was installed, the operation frequency and mode would be fixed, and could be changed only by dynamically allocated spectrum. A study [4] analyzed spectrum sensing of cognitive radio and proposed a joint detection algorithm based on energy detection and cyclostationary characteristics. It is used for spectrum sensing in mine communication. Previous studies [5, 6] suggested using Markov model to analyze dynamic spectrum access system based on overlay access method between a narrowband system and a broadband system. A three-dimensional Markov model was put forward in [7] to study the secondary users' performance in the dynamic spectrum access system giving priority access for authorized users. Another study [8] added handoff mechanism to analyze the performance of secondary users. The impact of dynamic spectrum access on secondary users' performance can be found in [9] and [10], which introduced whether the secondary users can have a new dynamic spectrum access scheme under buffer mechanism. In the above studies, the overlay dynamic spectrum access systems are the basis of the modeling and analysis. Researchers [11] studied the spectrum sharing performance analysis of cognitive users under interference temperature constraints. The three-dimensional Markov chain was used to model 
dynamic spectrum sharing strategy, and the sharing performance of cognitive users was analyzed.

Two kinds of users are supposed to exist in the cognitive radio study: authorized users and cognitive users. The spectrum resources give priority access to the priority access. Generally, the cognitive users can share the spectrum resources without affecting the authorized users' experience. The concept distinction between cognitive users and authorized users is relatively weak in coal mine wireless communication. The coal mine wireless signals can be divided into monitor signal, voice signal and video signal. They show different importance and numbers. The importance decreases from monitor signal, voice signal to video signal, and the numbers increase from monitor signal, voice signal to video signal. On the basis, the users in coal mines are further divided into primary users (PU), secondary users (SU) and tertiary users (TU), corresponding to monitor signal, voice signal and video signal respectively. The priority access level gradually decreases.

This paper studies the allocation model of underground wireless dynamic spectrum under interference temperature constraints. Considering the three kinds of users, firstly, multi-dimensional Markov chain model is used to model the dynamic spectrum sharing system under interference temperature constraints. Then, the performance of low priority users are analyzed in the system. Secondly, the performance of dynamic spectrum allocation system under interference temperature constraints and overlay dynamic spectrum access system is compared. Simulation experiment indicates that the performance of low priority users in the dynamic spectrum allocation system under interference temperature constrains is significantly better than that in overlay dynamic spectrum access system.

\section{SYSTEM MODELING}

\subsection{Scene Description}

The scene model is shown in Fig. (1). The primary users, secondary users and tertiary users are supposed to be Poisson arrival process, and the arrival rate is $\lambda_{p}, \lambda_{s}$ and $\lambda_{t}$ respectively. The service time indicates negative exponential distribution, and the average service time is $1 / \mu_{p}, 1 / \mu_{s}$ and $1 / \mu_{t}$ respectively. In the Fig. (1), the users arrival rate is indicated with the subscript $i, j \in\{p, s, t\}$.

The channel number in the system is set as N. The access of the primary users, secondary users and tertiary users all will not reject the service in real time. When the service is needed, the spare channels will provide access services directly. If there are no spare channels, the signal priority will be used to determine whether or not to block the service.

\subsection{Access Systems}

High priority users and low priority users are a one-toone correspondence. A single channel uses modified overlay or underway method to realize the channel sharing between users. The details are in the followings:
When the interference of low priority users to high priority users is below the interference temperature, the sharing channel can be realized.

(2) When there are spare channels in the system, the new users should access to a spare channel by the scheduler (spectrum access management) instead of the used channel. This can enhance the existing users' performance.

(3) If all the channels are taken, new users may be blocked. Whether or not to block the new users depends on two conditions. One is whether there is a channel meeting the conditions of interference temperature. The other is whether there is a channel for low priority users.

(4) Due to large data and long service time of voice signal and video signal, it is specified that the secondary users and tertiary users can't share the channels, i.e., channel sharing only happens between primary users and secondary users or between secondary users and tertiary users.

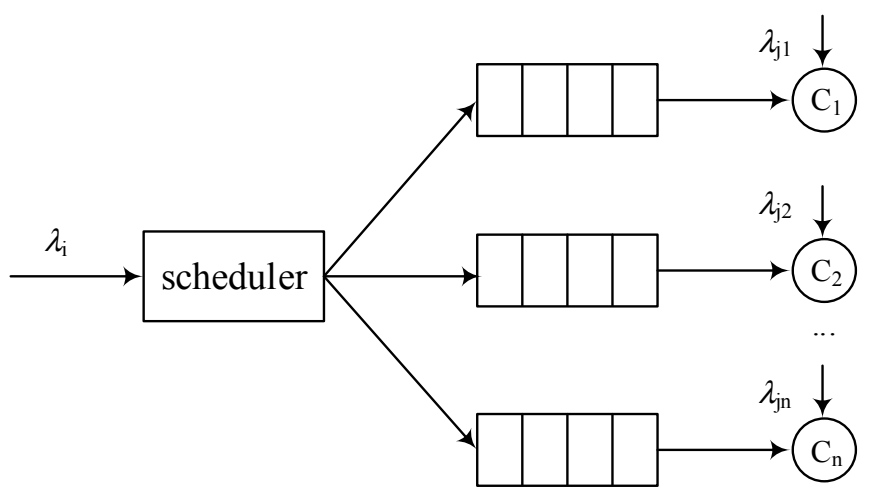

Fig. (1). Scene description.

\subsection{Access Flow}

Suppose that the probability of sharing channel satisfying interference temperature constraints for low priority users is $p$, when the $p$ is 0 , the dynamic spectrum access system is overlay access method. When the $p$ is 1 , the dynamic spectrum access system is underlay access method. Interrupting probability and blocking probability are defined to describe the performance of dynamic spectrum allocation model. Interrupting probability refers to the probability that the service of low priority has to be interrupted due to the arrival of high priority users in the channel. Blocking probability is the probability that shows the low priority users are blocked and can't access the channel because of non-availability of channels for the low priority users.

Fig. (2) shows the access flow for a new user. When there are spare channels in the system, the spectrum scheduler will randomly assign a spare channel for the new user. If there are no spare channels, the spectrum scheduler will randomly assign a channel for the new user and judge whether the interference temperature constraints conditions are satisfied or not.

The randomly assigned channel is other than the same channel of itself. The basic idea is to compare the using times and the merits of the channels. The channels with less 


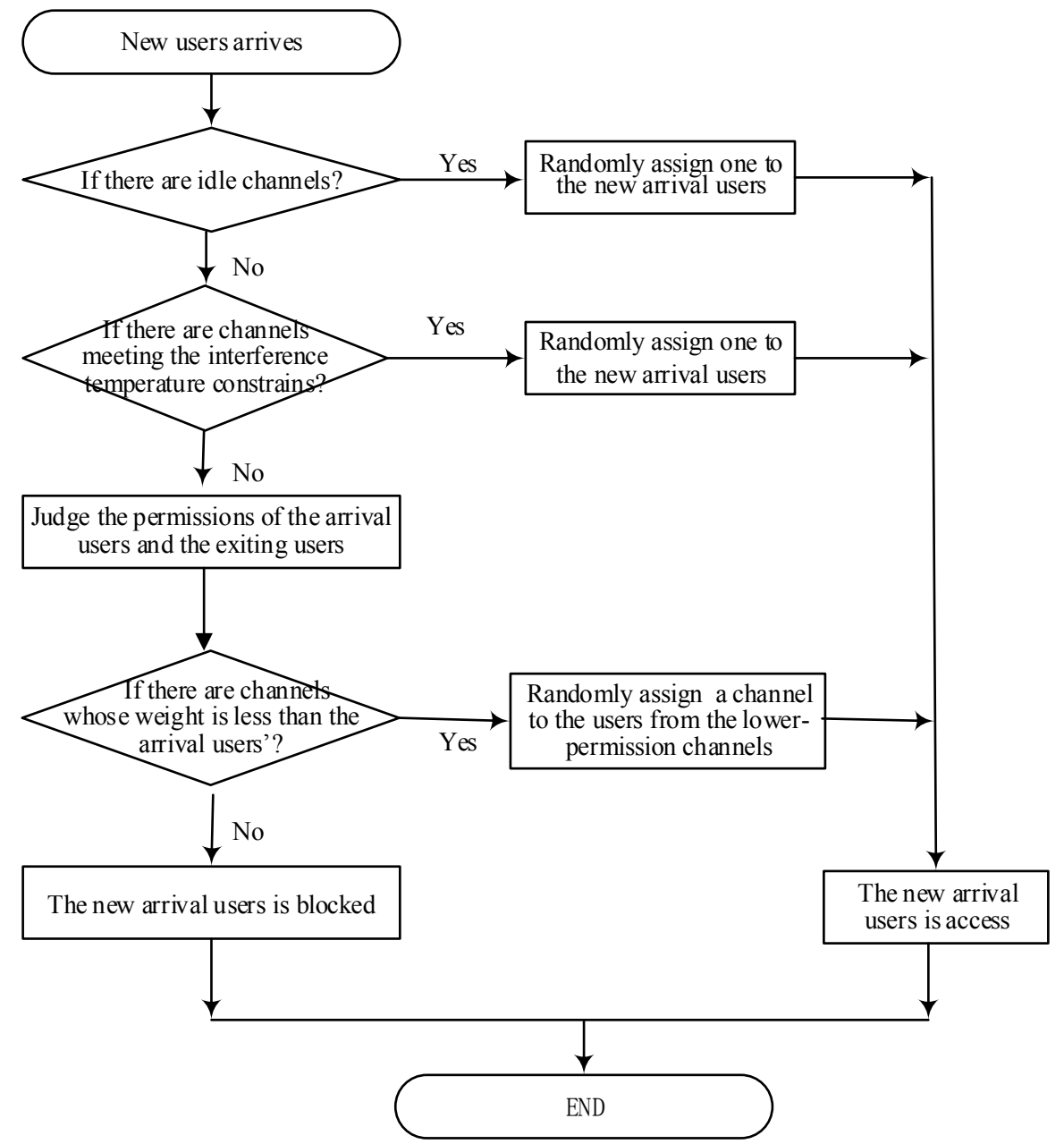

Fig. (2). New users access flow chart.

using times and small interference temperature can be chosen as the channels for the new users. When choosing the channels, the spectrum scheduler will generate a random number between 0 and 1 . Then, a value can be calculated. If the value is less than the set threshold, the channel cannot be the alternative channel.

\section{MODELING ANALYSIS}

\subsection{Discrete Time Markov Model}

Markov chain is a discrete-time stochastic process with characteristics of Markov. A Markov chain can form into a $\operatorname{triad}(S, P, Q)$, among them, $\mathrm{S}$ is the system state space, and

$i, j$ said state; $P=\left[P_{i j}\right]_{n \times n}$ is the system state transition probability matrix, $Q=\left[q_{1}, q_{2} \ldots q_{n}\right]$ is the initial probability distribution, satisfy $\sum_{i} q_{i}=1$.

A multi-dimensional Markov chain is used to model dynamic spectrum sharing system under interference temperature constraints. System state is expressed by $(i, j, k$, $m)$. $i$ represents the number of monitor signal occupying channels in the dynamic spectrum access system; $j$ represents the number of voice signal, $k$ represents the number of video signal, and $m$ represents the number of sharing channels. For better description, the number of sharing channels can be expressed by binary array, i.e., $m=\left(m_{1}, m_{2}\right) . m_{1}$ refers to the number of sharing channels between primary users and secondary users, and $m_{2}$ refers to the number of sharing channels between primary users and tertiary users. Obviously, they meet $m=m_{1}+m_{2}$. The system state space $\Gamma$ can be expressed by:

$$
\begin{aligned}
\Gamma=\{(i, j, k, m) \mid 0 \leq i \leq N, 0 \leq j \leq N, 0 \leq k \leq N, \\
\max (0, i+j+k-N) \leq m \leq \min [i, j+k]\}
\end{aligned}
$$

\subsection{System Events and State Transition Diagram}

System has the following six events: primary users' request arrival events, primary users' service completion events; secondary users' service completion events, secondary users' service completion events; tertiary users' arrival events, tertiary users' service completion events.

\subsubsection{Primary Users Request Arrival Events}

The channels are not all occupied by the primary users $(i<N)$.

If a primary user arrives, the channel access protocol will randomly select an access from the unoccupied channels for 
the new user. If there are other users in the selected channel (the probability is $(j+k-m) /(N-i)$ ), the interference temperature constraints will be based on judging whether the access of newly arrived primary user will influence the existing primary users quality of service. If the existing primary users quality of service is not affected (the probability is $p$ ), the newly arrived primary users can access and share the channel with the low priority users. The system state changes from $(i, j, k, m)$ to $(i+1, j, k, m+1)$. The transition number in per unit time is

$p \cdot \lambda_{p} \cdot(j+k-m) /(N-i)$.

If the primary users' service quality is affected by the service for low priority users (the probability is $(1-p)$ ), the service for low priority users will be interrupted and the primary user will access the channel. The system state will change from $(i, j, k, m)$ to $(i+1, j-1, k, m)$ or $(i+1, j, k-1, m)$

. Suppose $m_{1}$ and $m_{2}$ represent the number of sharing channels between primary users and secondary users, primary users and tertiary users respectively, then $\left(j-m_{1}\right) /(j+k-m)$ and $\left(k-m_{2}\right) /(j+k-m)$ respectively represent the proportion of secondary users' channels and tertiary users' channels (unoccupied by the primary users) in non-primary users' proportion in all channels.

When $q_{1}=\left(j-m_{1}\right) /(j+k-m)$ and $q_{2}=\left(k-m_{2}\right) /(j+k-m)$ $\left(q_{1}+q_{2}=1\right)$, the system state will change from $(i, j, k, m)$ to $(i+1, j-1, k, m)$ or $(i+1, j, k-1, m)$. The transition numbers will be $q_{1}(1-p) \cdot \lambda_{p} \cdot(j+k-m) /(N-i) \quad$ and $q_{2}(1-p) \cdot \lambda_{p} \cdot(j+k-m) /(N-i)$ respectively.

If there are no low priority users in the selected channel (the probability is $[N-i-(j+k-m)] /(N-i)$ ), the primary users will access directly. The system state will change from $(i, j, k, m)$ to $(i+1, j, k, m)$. The transition number will be $\lambda_{p} \cdot[N-i-(j+k-m)] /(N-i)$ in per unit time.

All the channels are occupied by primary users in the system $(i=N)$.

At this time, there are no available channels for the newly arrived primary users, so the new primary users will be blocked, and the system state is still $(i, j, k, m)$.

\subsubsection{Primary Users Service Completion Events}

No primary users in the system $(\mathrm{i}=\mathrm{m}=0)$

When there are no primary users in the system, "Primary users' service completion" is unlikely to happen.

The users in the channels only occupied by primary users will complete the service $(\mathrm{i}>0)$.

The service completion of primary users happens in the channels only occupied by primary users with a probability of $(i-m) / i$. The system state changes from $(i, j, k, m)$ to $(i-1, j, k, m)$. The transition number is $(i-m) \mu_{p}$ in per unit time.
Primary users service completion in the sharing channels by primary users and other users $(i>0, m>0)$.

The service completion of primary users happens in the sharing channels with a probability of $m / i$. The system state changes from $(i, j, k, m)$ to $(i-1, j, k, m-1)$. The transition number is $m \mu_{p}$ in per unit time.

\subsubsection{Secondary Users Request Arrival Events}

There are free channels in the system $(i+j+k-m<N)$.

If there are free channels in the system, the newly arrived secondary users will randomly access a free channel. The system state changes from $(i, j, k, m)$ to $(i, j+1, k, m)$. The transition number is $\lambda_{s}$ in per unit time.

There are no free channels in the system $(i+j+k-m=N)$.

The newly arrived users will randomly access a channel occupied by primary users. If the secondary user's interference to primary user is below the interference temperature threshold (the probability is $p$ ) and doesn't affect primary users' quality of service, the secondary user can access the channel. That is, when the new user arrives, the randomly pre-selected channel will be firstly considered instead of accessing the channel directly. The system state changes from $(i, j, k, m)$ to $(i, j+1, k, m+1)$. The transition number is $\lambda_{s} \cdot\left[1-(1-p)^{(i-m)}\right]$ in per unit time. If the interference temperature constraints are not satisfied, the secondary users need to judge whether or not the channels for low priority users (tertiary users) exist. If there are channels for low priority users $(k>0)$, the system state will change from $(i, j, k, m)$ to $(i, j+1, k-1, m)$. The transition number is $\lambda_{s} \cdot(1-p)^{(i-m)} \cdot\left(k-m_{2}\right) / k$ in per unit time, among which, $m_{2}$ represents the number of sharing channels by primary users and tertiary users. If there are no channels for low priority users $(k=0)$, the arrived user will be blocked, and the system state $(i, j, k, m)$ will be unchanged.

\subsubsection{Secondary Users Service Completion Events}

There are no secondary users in the system $(\mathrm{j}=0)$.

There are no secondary users in the system, so the secondary users service completion events are unlikely to happen.

Secondary users service completion events happen in the channels only when occupied by secondary users $(j>0)$.

Secondary users service completion events happen in the channels only when occupied by secondary users (the probability is $\left(j-m_{1}\right) / j, m_{1}$ is the number of sharing channels by primary users and tertiary users). The system state changes from $(i, j, k, m)$ to $(i, j-1, k, m)$. The transition number is $\left(j-m_{1}\right) \mu_{s}$ in per unit time.

Secondary users service completion events happen in the sharing channel by primary users and secondary users $(j>0$, $\mathrm{m}>0$ ). 
Secondary users service completion events happen in the sharing channel by primary users and secondary users (the probability is $\left.m_{1} / j\right)$. The system state changes from $(i, j, k, m)$ to $(i, j-1, k, m-1)$. The transition number is $m_{1} \mu_{s}$ in per unit time.

\subsubsection{Tertiary Users Arrival Events}

There are free channels in the system $(i+j+k-m<N)$.

When the free channels are available in the system, similar to the secondary users' arrival events, the newly arrived tertiary user will randomly access a free channel. The system state changes from $(i, j, k, m)$ to $(i, j, k+1, m)$. The transition number is $\lambda_{t}$ in per unit time.

There are no free channels in the system $(i+j+k-m=N)$.

There are channels only occupied by primary users in the system $(i>m)$.

The new user will randomly access a channel only occupied by primary users. The tertiary user can access the channel if the interference is below interference temperature threshold (the probability is $p$ ) and doesn't affect the primary users' quality of service. The transition number is $\lambda_{t} \cdot\left[1-(1-p)^{(i-m)}\right]$ in per unit time. Otherwise, if the tertiary user's access affects the primary users' quality of service (the probability is $(1-p)$ ), the tertiary user will be blocked, and the system state is still $(i, j, k, m)$.

There are no free channels only occupied by primary users in the system $(i=m)$.

When there are no free channels only occupied by primary users in the system, the new user's service will be blocked, and the system state $(i, j, k, m)$ will be unchanged.

\subsubsection{Tertiary Users Service Completion Events}

No tertiary users in the system $(k=0)$.

There are no tertiary users in the system, so the tertiary users service completion events are unlikely to happen.

Tertiary users service completion in the channels only occupied by tertiary users $(k>0)$.

Tertiary users service completion events happen in the channels only when occupied by tertiary users (the probability is $\left.\left(k-m_{2}\right) / k\right)$. The system state changes from $(i, j, k, m)$ to $(i, j, k-1, m)$. The transition number is $\left(k-m_{2}\right) \mu_{t}$ in per unit time.

Tertiary users service completion in the sharing channel happens by primary users and tertiary users $(k>0, m>0)$.

When the tertiary users service completion happens in the sharing channel (the probability is $m_{2} / k$ ), the system state will change from $(i, j, k, m)$ to $(i, j, k-1, m-1)$. The transition number is $m_{2} \mu_{t}$ in per unit time.

According to the above analysis, the users' arrival events have two different cases: when the free channels are available, i.e., $i+j+k-m<N$, a new user will access a free channel directly, the system state transition diagram is shown in Fig. (3a); when there are no free channels in the system, i.e., $i+j+k-m=N$, the system state depends on new user's authority. If the user authority is high, the system state will change. Otherwise, the system will remain unchanged. The system state transition diagram is shown in Fig. (3b).

(a) $i+j+k-m<N$

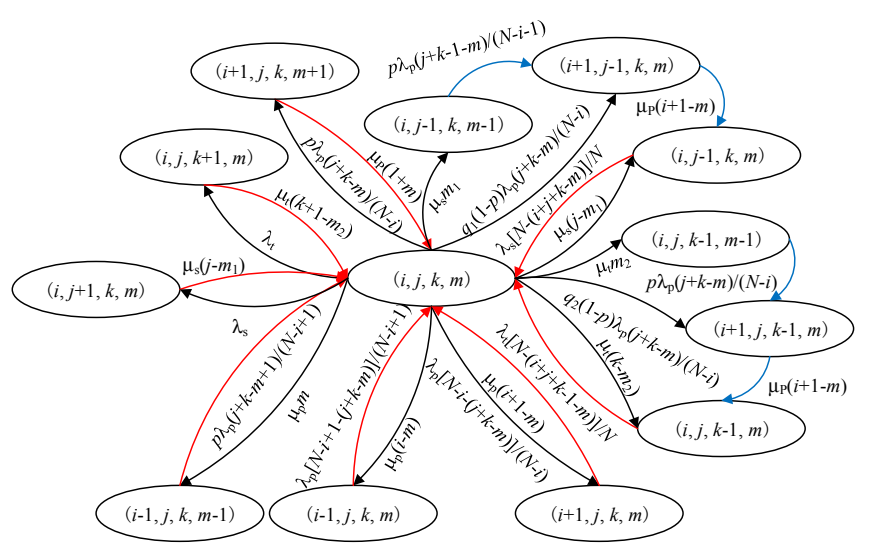

(b) $i+j+k-m=N$

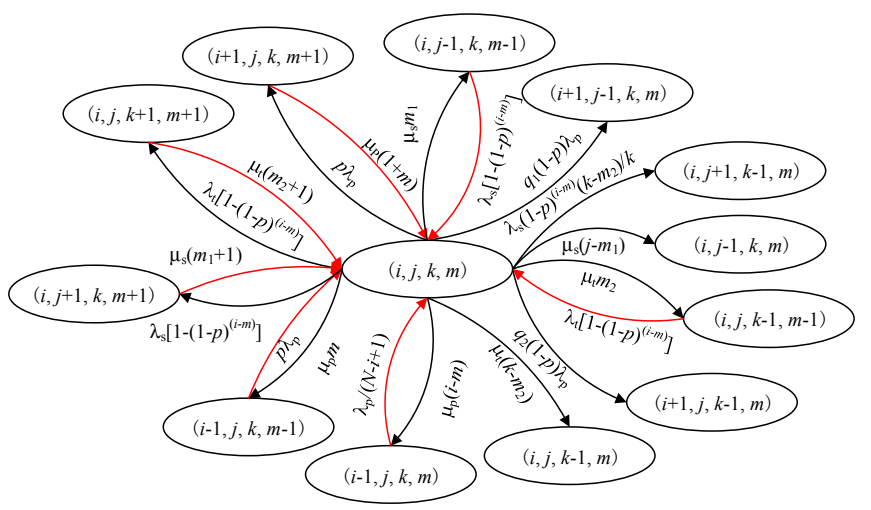

Fig. (3). System state transition diagram.

\subsection{State Equation}

$P(i, j, k, m)$ is defined as the steady-state probability of system state $(i, j, k, m)$. In the system state transition diagram, the input stream is equal to the output stream at steady state. On this basis, the probability equation in different states can be obtained.

When there are free channels in the system, i.e., $i+j+k-m<N$

$$
\begin{aligned}
& \left(\lambda_{p}+\lambda_{\mathrm{s}}+\lambda_{t}+\mu_{p} i+\mu_{\mathrm{s}} j+\mu_{\mathrm{t}} k\right) \cdot P(i, j, k, m) \\
& =\lambda_{s}[N-(i+j+k-m)] / N \cdot P(i, j-1, k, m) \\
& \quad+\lambda_{t}[N-(i+j+k-1-m)] / N \cdot P(i, j, k-1, m) \\
& \quad+\lambda_{p}[N-i+1-(j+k-m)] /(N-i+1) \cdot P(i-1, j, k, m) \\
& \quad+p \lambda_{p}(j+k-m+1) /(N-i+1) \cdot P(i-1, j, k, m-1) \\
& \quad+\mu_{p}(i+1-m) \cdot P(i+1, j, k, m)+\mu_{p}(1+m) \cdot P(i+1, j, k, m+1) \\
& \quad+\mu_{s}\left(j+1-m_{1}\right) \cdot P(i, j+1, k, m)+\mu_{t}\left(k+1-m_{2}\right) \cdot P(i, j, k+1, m)
\end{aligned}
$$

When there are no free channels in the system, i.e., 
$i+j+k-m=N$

$\left\{\lambda_{p}+\lambda_{\mathrm{s}}\left[1-(1-p)^{i-m} m_{2} / k\right]+\lambda_{t}\left[1-(1-p)^{i-m}\right]\right.$

$\left.+\mu_{p} i+\mu_{\mathrm{s}} j+\mu_{\mathrm{t}} k\right\} \cdot P(i, j, k, m)$

$=\lambda_{s}\left[1-(1-p)^{i-m}\right] \cdot P(i, j-1, k, m-1)$

$+\lambda_{t}\left[1-(1-p)^{i-m}\right] \cdot P(i, j, k-1, m-1)$

$+\lambda_{p}^{t} /(N-i+1) \cdot P(i-1, j, k, m)$

$+p \lambda_{p} \cdot P(i-1, j, k, m-1)+\mu_{p}(1+m) \cdot P(i+1, j, k, m+1)$

$+\mu_{s}\left(1+m_{1}\right) \cdot P(i, j+1, k, m+1)+\mu_{t}\left(1+m_{2}\right) \cdot P(i, j, k+1, m+1)$

The sum of all steady-state probability constraints need to satisfy

$$
\sum_{(i, j, k, m) \in \Gamma} P(i, j, k, m)=1
$$

In this way, equation set can change into two matrix equations: $P=P Y$ and $P e=1 . P$ is steady-state probability vector, $Y$ is the transmission matrix and $e$ is column vector as all elements are 1. Due to the large system state space, iterative algorithm can be used to solve linear system of equations.

\section{Specific process is:}

(1) According to the state space, structure matrix $Y$;

(2) Set the iteration convergence criterion error $\varepsilon$, relaxation factor $\omega, 1 \leq \omega<2$;

(3) Set the initial state probability $P^{(0)}$;

(4) Calculate $P^{(n)}=\omega P^{(n-1)} Y+(1-\omega) P^{(n-1)}$, Until meet the following inequality

$\sum_{y \in \Gamma} \mathrm{l} \frac{P_{y}^{(n)}-P_{y}^{(n-1)}}{P_{y}^{(n)}+P_{y}^{(n-1)}} \mathrm{K}<\varepsilon$

Finally concluded the steady state probability $P$.

\subsection{Performance Analysis}

If any channel occupied by primary users doesn't satisfy the interference temperature constraints in the system, the newly arrived low priority users can't access the channel and will be blocked. Set the blocking probability of the new arrival users is $P_{B}$,

$P_{B}=\sum_{(i, j, k, m) \in \Gamma, i+j+k-m=N} P(i, j, k, m) \cdot \lambda_{s} \cdot(1-p)^{i-m}$

The interception probability of the secondary users and the tertiary users is $P_{I}$. When the system state $(i, j, k, m)$ is in the moment of the primary users arriving, the interception probability of the secondary users is

$P_{I S}=\sum_{(i, j, k, m) \in \Gamma} P(i, j, k, m) \cdot \lambda_{p} \cdot(1-p) \cdot\left(j-m_{1}\right) /(N-i)$

The interception probability of the tertiary users is

$P_{I T 1}=\sum_{(i, j, k, m) \in \Gamma} P(i, j, k, m) \cdot \lambda_{p} \cdot(1-p) \cdot\left(k-m_{2}\right) /(N-i)$

When the system state $(i, j, k, m)$ is at the interception of the secondary users arriving, the interception probability of the tertiary users is:

$$
P_{I T 2}=\sum_{(i, j, k, m) \in \Gamma, i+j+k-m=N} P(i, j, k, m) \cdot \lambda_{s} \cdot(1-p)^{i-m} \cdot\left(k-m_{2}\right) / k
$$

Above all

$$
\begin{aligned}
P_{I} & =P_{I S}+P_{I T 1}+P_{I T 2} \\
& =\sum_{(i, j, k, m) \in \Gamma} P(i, j, k, m) \cdot \lambda_{p} \cdot(1-p) \cdot(j+k-m) /(N-i) \\
& +\sum_{(i, j, k, m) \in \Gamma, i+j+k-m=N} P(i, j, k, m) \cdot \lambda_{s} \cdot(1-p)^{i-m} \cdot\left(k-m_{2}\right) / k
\end{aligned}
$$

\section{SIMULATION TEST}

This part focuses on users' performance in the dynamic spectrum access system under interference temperature constraints. When the dynamic spectrum under interference temperature constraint access to the system, $p$, is not 0 ; When access by the Overlay mode, $p$ is 0 .

(1) The interrupting probability $P_{I}$ and blocking probability $P_{B}$ 's variation with primary users' arrival rate $\lambda_{p}$

Set $N=3, \lambda_{s}=\lambda_{t}=0.15, \mu_{p}=\mu_{s}=\mu_{t}=0.2, p=0.4$, the simulation figure is shown in Fig. (4).

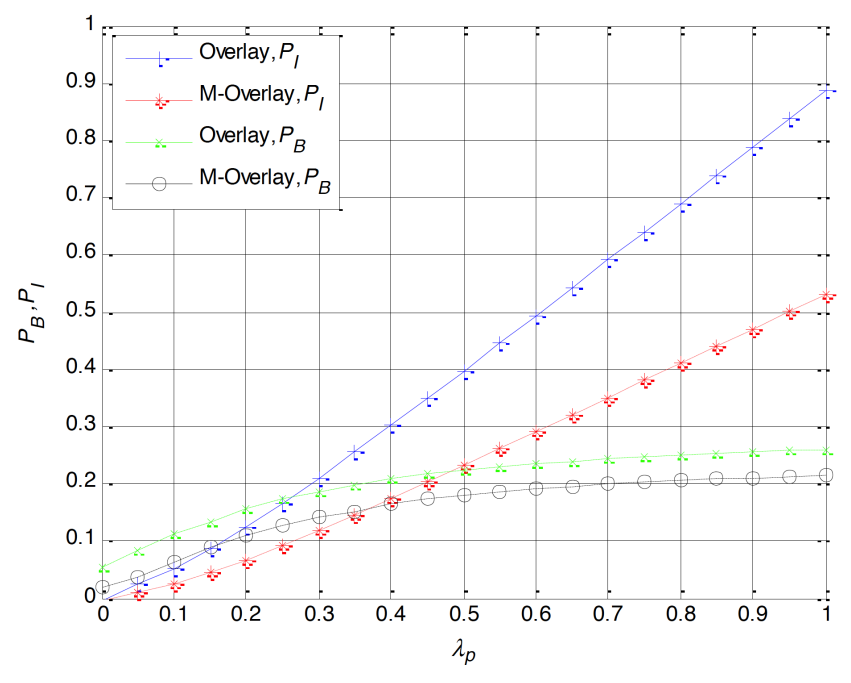

Fig. (4). $P_{I}, P_{B}$ with $\lambda_{p}$.

Fig. (4) indicates that the users interrupting probability increases linearly with the increase of primary users arrival rate, and the blocking probability firstly increases and then keeps steady increase. The interrupting probability increases with more primary users accessing, and the running low priority users' transmission is also frequently interrupted. The initial interrupting probability is 0 , because there is no primary users' arrival. With the increasing primary users, the average service time is unchanged. The channels are increasingly occupied by primary users and other users blocking probability is increasing.

(2) The interrupting probability $P_{I}$ and blocking probability $P_{B}$ 's variation with secondary users' arrival rate $\lambda_{s}$. 
Set $N=3, \lambda_{p}=0.3, \lambda_{t}=0.15, \mu_{p}=\mu_{s}=\mu_{t}=0.2, p=0.4$, the simulation figure is shown in Fig. (5).

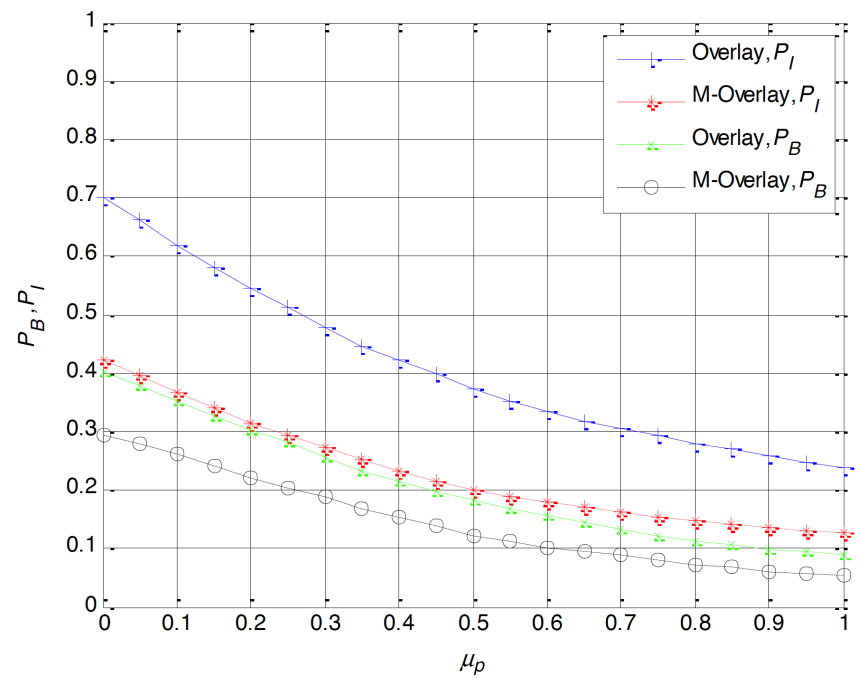

Fig. (5). $P_{I}, P_{B}$ with $\lambda_{s}$ set.

Fig. (5) indicates that the users interrupting probability flattens out basically with the increase of primary users arrival rate, and the blocking probability increases linearly.

(3) The variation of the interception probability $P_{I}$ and the blocking probability $P_{B}$ varied with the completion rate of the primary users $\mu_{p}$.

Set $N=3, \lambda_{p}=0.6, \lambda_{s}=\lambda_{t}=0.2, \mu_{s}=\mu_{t}=0.6, p=0.4$, the simulation figure is shown in Fig. (6).

Fig. (6) shows that, with the increase of primary users completion rate, the interrupting probability and blocking probability gradually decrease. It is because, with the increase of primary users' completion rate, the average service time decreases and more channels become available for primary and other users. Thus, other users interrupting probability and blocking probability will decrease. When primary users' service completion rate $\mu_{p}$ exceeds a certain value, and there are enough channels in the system for other users to access and transmit, then the interrupting probability and blocking probability decrease gradually. In the figure, the initial state determines the initial interrupting probability and blocking probability.

(4) The variation of the interception probability $P_{I}$ and the blocking probability $P_{B}$ varied with the completion rate of the secondary users $\mu_{s}$.

Set $N=3, \lambda_{p}=0.6, \lambda_{t}=0.2, \mu_{p}=\mu_{t}=0.6 p=0.4$, the simulation figure is shown in Fig. (7).

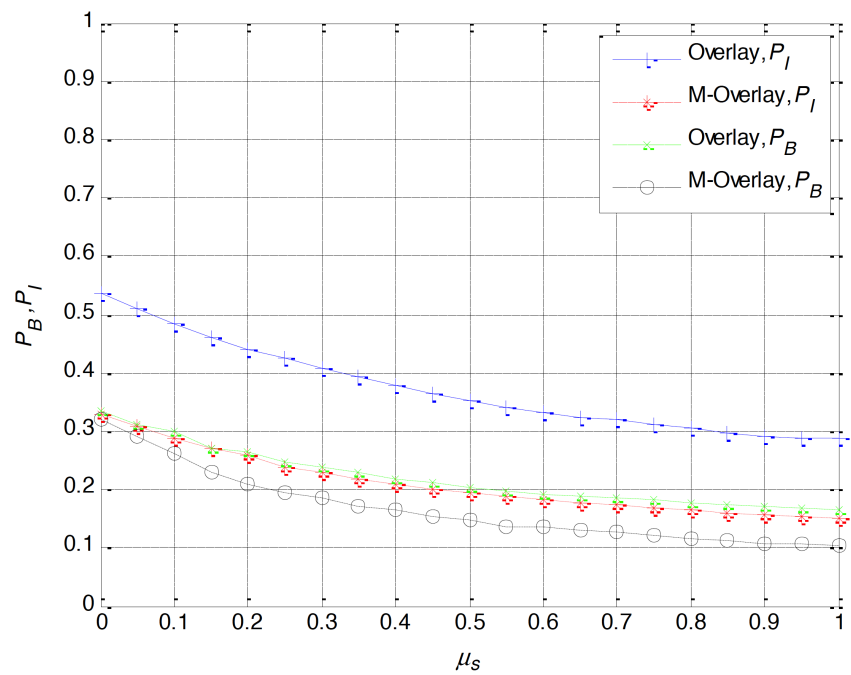

Fig. (6). $P_{I}, P_{B}$ with $\mu_{p}$.

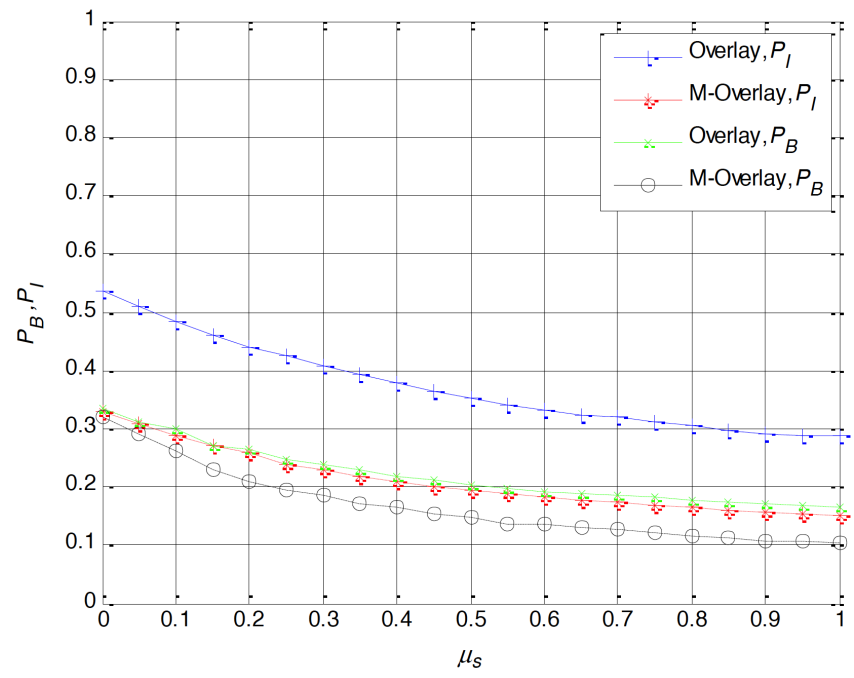

Fig. (7). $P_{I}, P_{B}$ with $\mu_{p}$.

Fig. (7) shows that, with the increase of the completion rate of the secondary users, the interception probability $P_{I}$ and the blocking probability $P_{B}$ gradually decrease, the duration time of the secondary users service in system reduces, and more channels are available for the access of the primary users and other users.

The variation of the interception probability $P_{I}$ and the blocking probability $P_{B}$ varied with the probability of channel sharing $p$.

Fig. (8) shows that, with the increase of the channel sharing probability $p$, the interrupting probability and blocking probability gradually decrease. When $p$ is 1 , the interception probability is 0 . The increase of $p$ means that more users can successfully have access to channels for sharing with the primary users, and other users' interception decreases. When $p$ is 1 , there are no other users waiting in system, and the interception probability is 0 . 


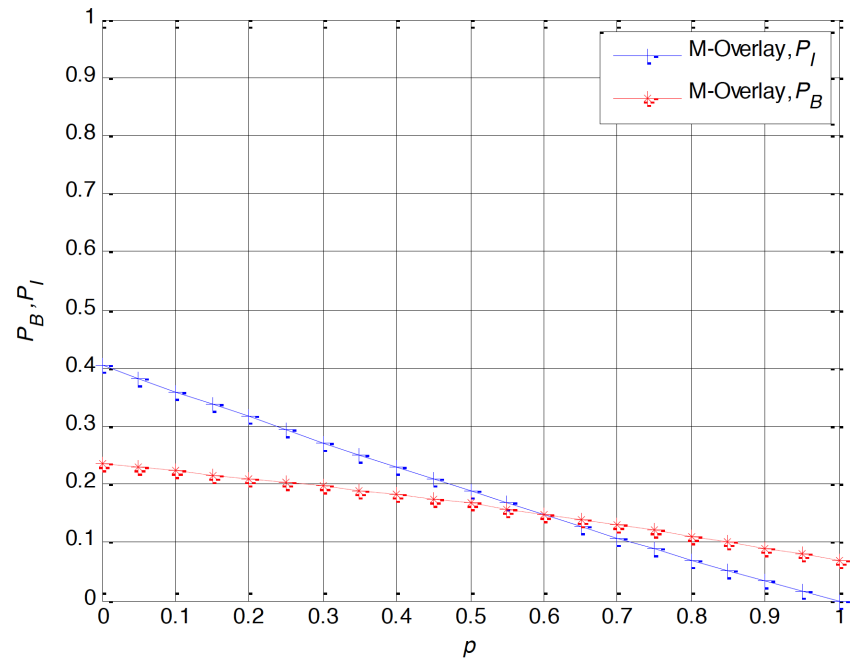

Fig. (8). $P_{I}, P_{B}$ with $\mu_{s}$.

(6) System performance variation with primary users' arrival rate under channels number changing conditions

Simulation results can be seen in Fig. (9). With the increase of channel numbers, the interrupting and blocking probability decreases. That is, as the number of channels increases, more users can access the channels and the system performance will be improved.

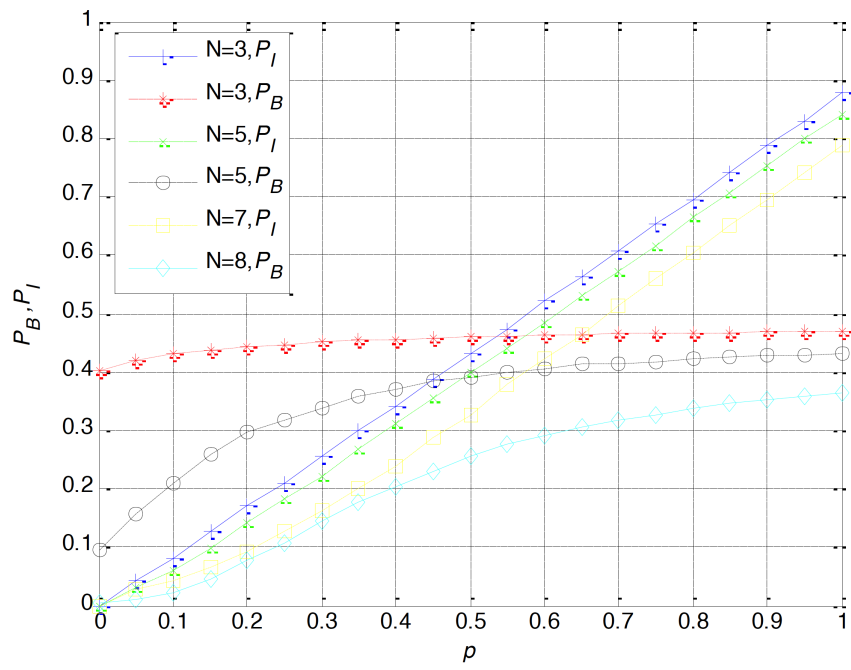

Fig. (9). $P_{I}, P_{B}$ with $\lambda_{p}$ and $N$.

It can be seen from the experiment simulation that under the same parameters, the performance of the modified MOverlay sharing access system is better than that of the Overlay access system with the increase of authorized users and cognitive users' arrival rate and completion rate.

\section{CONCLUSION}

The concept distinction between cognitive users and authorized users is relatively weak in coal mine wireless communication. The coal mine wireless signals can be divided into monitor signal, voice signal and video signal. They show different importance and numbers. The importance decreases from monitor signal, voice signal to video signal, and the numbers increase from monitor signal, voice signal to video signal. On the basis, the dynamic spectrum allocation model is designed for the coal mine wireless communication. The design idea that the users in coal mines can be divided into primary users (PU), secondary users (SU) and tertiary users (TU) is proposed. The spectrum sharing access system is designed and specifies that the sharing can only happen between primary users and secondary users, and primary users and tertiary users.

In order to describe three kinds of users' dynamic spectrum allocation mechanism, a multi-dimensional Markov chain is used to model the dynamic spectrum allocation system under inference temperature constrains. Six events and system state transitions are introduced in detail and the transition number in per unit time is calculated. State transition diagrams in the cases of free channels $i+j+k-m<N$ and no free channels $i+j+k-m=N$ are designed respectively, and the state transition formulas are given. State equation and iteration solution algorithm are used to deduce the index formulas (blocking probability formula and interrupting probability formula) to measure sharing performance by low priority users. Finally, we analyze the variation of performance indexed in different state and compare the performance of dynamic spectrum allocation system under interference temperature constrains and overlay dynamic spectrum access system. The simulation experiment indicates that the performance of low priority users in the dynamic spectrum allocation system under interference temperature constrains is significantly better than that in overlay dynamic spectrum access system, presenting more reasonable users division and superior dynamic spectrum allocation model.

\section{CONFLICT OF INTEREST}

The authors confirm that this article content has no conflict of interest.

\section{ACKNOWLEDGEMENTS}

This work was financially supported by Sub-project under National Science and Technology Support Program (2012BAH12B01).

\section{REFERENCES}

[1] Shen, Z.; Hu, Z.X. Comments on sensory mine internet of things and mine comprehensive automation. Coal Sci. Technol., 2012, 40, 83-86.

[2] Shen, Z.; Jie, D.E.; Zhao, X.; Gang, H. Part III of lecture of internet of things and sensor mine - characteristics and key technologies of sensor mine internet of things industry and mine automation. Ind. Mine Automat., 2010, 12, 117-121.

[3] Zhen, C.G.; Jie, D.E.; Shen, Z.; Song, H.Q. Brief analysis of application of cognitive radio in mine communication. Ind. Mine Automat., 2009, 6, 12-14.

[4] Zhen, C.G.; Jie, D.E.; Wei, Q.H. Research of spectrum sensing in coal mine based on cognitive radio. Video Appl. Project, 2009, 33(12), 92-94.

[5] Akyildiz, I.F.; Lee, W.Y.; Vuran, M.C.; Mohanty, S. Next generation/dynamic spectrum access/cognitive radio wireless networks: A survey. Comput. Netw., 2006, 50(13), 2127-2159. 
[6] Yan Z. Dynamic Spectrum Access in Cognitive Radio Wireless Networks. IEEE International Conference on Communications in 2008, Beijing, 2008, pp. 4927-4392.

[7] Haykin, S. Cognitive radio: brain-empowered wireless communications. IEEE J. Sel. Areas Commun., 2005, 23(2), 201220.

[8] Zhu, X.; Shen, L.; Yum, T.S.P. Analysis of cognitive radio spectrum access with optimal channel reservation. IEEE J. Sel. Areas Commun., 2006, 24, 626-636.

[9] Mitola, J. Cognitive radio: making software radios more personal. IEEE Pers. Commun., 1999, 6(4), 13-18.
[10] Menon, R.; Buehrer, R.M.; Reed, J. Outage Probability Based Comparison of Underlay and Overlay Spectrum Sharing Techniques. ${ }^{\text {st }}$ IEEE International Symposium on New Frontiers in Dynamic Spectrum Access Networks, 2005, pp. 101-109.

[11] Yan, Z.; Zhang, X.; Wang, W.B. Performance Analysis of Secondary Users in Dynamic Spectrum Access Under Interference Temperature Constraints. In: $10^{\text {th }}$ IEEE International Conference on Computer and Information Technology (CIT 2010), Bradford, 2010, pp. 2655-2661.

(C) Guizhen et al.; Licensee Bentham Open.

This is an open access article licensed under the terms of the Creative Commons Attribution Non-Commercial License (http://creativecommons.org/licenses/by-nc/4.0/) which permits unrestricted, non-commercial use, distribution and reproduction in any medium, provided the work is properly cited. 